\title{
Desarrollo y validación de la Escala de Convivencia Escolar (ECE)
}

The Development and Validation of the Schoolwide Climate Scale

Recepción: 14 Febrero 2014 | Aprobación: 23 Enero 2017

\author{
Rosario Del Rey \\ Universidad de Sevilla, España \\ ORCID: http://orcid.org/0000-0002-1907-5489 \\ José Antonio Casas \\ Universidad de Córdoba, España \\ ORCID: http://orcid.org/0000-0002-0272-1835 \\ Rosario Ortega Ruiz \\ Universidad de Córdoba, España \\ ORCID: http://orcid.org/0000-0003-2110-6931
}

a Autor de correspondencia. Correo electrónico:
delrey@us.es

Para citar este artículo: Del Rey, R., Casas, J. A., \& Ortega Ruiz, R. (2017). Desarrollo y validación de la Escala de Convivencia Escolar (ECE). Universitas Psychologica, 16(1), 1-11. http://dx.doi.org/10.11144/J averiana.upsy16-1.dvec

\section{RESUMEN}

En este estudio, se desarrolla y valida la Escala de Convivencia Escolar que integra gran parte de las perspectivas existentes en la literatura científica sobre este tema. El principal objetivo de la investigación fue contrastar empíricamente la validez del constructo. La muestra estuvo compuesta por 3146 estudiantes de educación primaria y secundaria obligatoria de Andalucía (España). Mediante análisis factoriales exploratorios y confirmatorios, se contrastó la idoneidad de un modelo explicativo de la convivencia escolar compuesto por ocho dimensiones. El resultado pone de manifiesto la importancia en el constructo de la gestión y el ejemplo de buenas relaciones que el profesorado ofrece al alumnado, así como la necesidad de incluir en él aspectos positivos y negativos de la misma.

Palabras clave

convivencia escolar; validación; primaria; secundaria.

\section{ABSTRACT}

The purpose of this study is the development and validation of the Schoolwide Climate Scale by integrating the most relevant existing perspectives described in scientific literature. The main objective of the investigation has been the empiric test of the validity of the construct. The sample consisted in 3,146 pupils of primary and secondary compulsory education in Andalusia, Spain. We have contrasted, through exploratory and confirmatory factor analysis, the suitability of an explanatory model of the school life composed of eight dimensions. The results show the relevant effect on the construct of the interpersonal management and the example given by the teachers to the pupils as regards good relations, as well as of the need of including its positive and negative aspects in the construct.

Keywords

school climate; schoolwide; validation; primary; secondary. 
El llamado Informe Delors (1996) ofreció una aproximación al concepto de "aprender a convivir" como uno de los cuatro objetivos de la educación para todos los países y todas las culturas. Esta meta se ha convertido en uno de los objetivos más importantes entre los fines de los diferentes sistemas educativos a nivel mundial. En España y países latinoamericanos, el nuevo constructo conocido como "convivencia escolar" ha adquirido un cierto protagonismo en la cultura y las prácticas educativas y se encuentra entre los conceptos más mencionados en el ámbito psicoeducativo (García Raga, \& López Martín, 2011). Un concepto definido como poliédrico y multidisciplinar (Godás Otero, Santos Rego, \& Lorenzo, 2008; Ortega \& Del Rey, 2004) que incluye elementos que van desde la vida social en la escuela y las aulas (Gázquez, Pérez, \& Carrión, 2011; Grisaleña, Fernández, \& Campo, 2005; Viguer \& Avià, 2009) y los conflictos y problemas de disciplina escolares que emergen en los procesos instruccionales (Caso, Salgado, Rodríguez, Contreras, \& Urías, 2010) hasta el más global de "clima escolar" (Fullan \& Hargreaves, 1992; Kuperminc, Leadbeater, Emmons, \& Blatt, 1997; Moos \& Moos, 1978).

En la esfera internacional, no existe una denominación homóloga a la expresión española convivencia, con la que referirse a ese amplio conjunto de elementos que la comunidad escolar hispana considera que están presentes en ella. Pero recientemente, algunos trabajos de investigación se refieren al clima escolar, desde una perspectiva de escuela global (schoolwide) que podríamos considerar homóloga (Burke et al., 2012; McIntosh et al., 2011). Trabajos científicos realizados desde esta perspectiva suelen focalizar el análisis no solo del alumnado, sino también del profesorado, las familias y las relaciones entre todos los agentes educativos e incluso comunitarios (Bear, Gaskins, Blank, \& Chen, 2011; Mitchell, Bradshaw, \& Leaf, 2010). El núcleo del concepto escuela global, como el de convivencia, focaliza las relaciones interpersonales que están siempre presentes en las tareas y los procesos relacionales que despliegan los agentes educativos y sus pupilos, cuando realizan su labor educativa (Ortega \& Del Rey, 2004). Sin embargo, no existen instrumentos rigurosamente validados para el concepto español de convivencia escolar y los instrumentos de clima escolar no incluyen esta consideración global de la convivencia (Bradshaw, Debnam, Koth, \& Leaf, 2009).

Partiendo del análisis del clima escolar, una de las escalas más utilizadas es la Escala de Clima Social en el Centro Escolar (CES) de Moos y Trickett (1974), que valora cuatro dimensiones: relaciones sociales, autorrealización, estabilidad y cambio. Otra escala muy utilizada es el School Development Program School Climate Survey (Haynes, Emmons, \& Ben-Avie, 1997), que considera ocho dimensiones significativas en el clima escolar: equidad y justicia, orden y disciplina, la participación de la familia, el intercambio de recursos, las relaciones interpersonales de los estudiantes, las relaciones maestro-alumno, la motivación para el logro y el edificio de la escuela. En recientes estudios (Bear et al., 2011), en la Delaware School Climate Survey-Student, se definen cinco factores en el constructo clima escolar: relaciones profesor-alumnado, relaciones entre el alumnado, equidad de las normas, seguridad en la escuela y problemas de violencia y aspectos afectivo-emocionales. Este instrumento es particularmente valioso a este respecto, al estar validado con población de varios países (Yang et al., 2013).

En el contexto español y latinoamericano, referido propiamente al constructo convivencia, se han utilizado muchos instrumentos que la han evaluado, considerando diferentes dimensiones (Ortega, Del Rey, \& Sanchez, 2012). Las dimensiones y factores señaladas en la literatura científica son: la calidad de las relaciones interpersonales en el centro educativo, la participación de las familias en el mismo, la naturaleza y gestión de las normas, la percepción de existencia de conflictos y problemas de indisciplina, disruptividad y violencia y las medidas educativas por desarrollar. Otros factores que se incluyen hacen referencia a problemas que alteran la convivencia como por ejemplo indisciplina, acoso escolar, conductas 
antisociales, disruptivas y de desmotivación y desinterés académico (Gazquez, Cangas, PerezFuentes, Padilla, \& Cano, 2007).

En síntesis, los instrumentos de medida del constructo convivencia no han llegado a establecer una homogeneidad razonable sobre cuáles son los factores que lo componen. En este sentido, es importante señalar que cualquier aproximación a una definición algo más operativa debe asumir que hay en esta tarea un importante reto de dificultad relevante, ya que la convivencia es, en gran medida, el resultado de la percepción que los protagonistas de la misma tienen sobre procesos cargados de subjetividad y de las propias experiencias del sujeto. Además, la subjetividad de la experiencia vivida debe ser considerada un factor interviniente en cualquier sistema de medida y valoración de la convivencia (Ortega \& Del Rey, 2004). Una aproximación a la objetivación del concepto debe aceptar que incluso la más fidedigna medida implica reconocer que hablamos de una construcción social (Bibou-Nakou, Tsiantis, Assimopoulos, Chatzilambou, \& Giannakopoulou, 2012).

En España, el último informe del Observatorio Estatal de la Convivencia Escolar realiza un primer intento de integración de las distintas aportaciones sobre la convivencia escolar y se definen por separado los indicadores globales de la convivencia escolar y los de los problemas que la afectan (Martín-Babarro, Martínez-Arias, \& Díaz-Aguado, 2010). Pero queda aún pendiente la confirmación empírica del constructo teórico que se ha ido construyendo a lo largo de la trayectoria de estudios de convivencia escolar, mediante el uso de un instrumento de medida riguroso y ajustado al heurístico. De esta forma, se delimitarían los aspectos más relevantes en la definición del constructo (Morata-Ramírez \& Holgado-Tello, 2013).

Por ello, el propósito de la investigación que aquí se presenta ha sido validar una herramienta que posibilite recoger, de la forma más parsimoniosa posible, los elementos que componen la convivencia escolar y establecer las dimensiones de la misma, tanto las positivas como las de los fenómenos que la perturban. Así mismo, pretende analizar su idoneidad para el análisis de la convivencia escolar entre el alumnado de educación primaria y el de educación secundaria, ya que ambas etapas educativas han sido diferenciadas en gran cantidad de estudios (Mitchell et al., 2010).

\section{Método}

\section{Participantes}

En la presente investigación, participó un total de 3146 sujetos (49\% chicas), escolarizados en 14 centros de Educación Secundaria Obligatoria (ESO) y 11 centros de Educación Primaria de todas las provincias de Andalucía, seleccionados por muestreo intencional dirigido o de conveniencia. Las edades de los sujetos estuvieron comprendidas entre 11 y 19 años $(M=14.21$; $D T=1.79)$. Siguiendo las recomendaciones para la validación de cuestionarios y escalas (Hair, Black, Babin, Anderson, \& Tatham, 2005). La muestra original se dividió en dos submuestras, muestra piloto y muestra validación, controlando la distribución de sexo y curso (Tabla 1 ).

TABLA 1

Descripción de las submuestras

\begin{tabular}{|c|c|c|c|c|c|c|}
\hline \multirow[b]{2}{*}{ Curso } & \multicolumn{2}{|c|}{ Muestra Piloto } & \multirow[b]{2}{*}{$\begin{array}{l}\text { Total } \\
n(\%)\end{array}$} & \multicolumn{3}{|c|}{ Muestra validación } \\
\hline & $\begin{array}{l}\text { on } \\
n(\%)\end{array}$ & $\begin{array}{l} \\
n(\%)\end{array}$ & & $\begin{array}{l}\hat{0} \\
n(\%)\end{array}$ & $\begin{array}{l}+ \\
n(\%)\end{array}$ & $\begin{array}{l}\text { Total } \\
n(\%)\end{array}$ \\
\hline $5 .^{\circ} \mathrm{P}$ & $26(5.5)$ & $19(4)$ & $45(9.6)$ & $141(5.3)$ & $123(4.6)$ & $264(9.9)$ \\
\hline $6^{\circ} \mathrm{P}$. & $22(4.7)$ & $30(6.4)$ & $52(11)$ & $145(5.4)$ & $152(5.7)$ & $297(11.1)$ \\
\hline $1 .^{\circ} \mathrm{ESO}$ & 49 (10.4) & $40(8.5)$ & $89(18.9)$ & $264(9.9)$ & $258(9.7)$ & $522(19.5)$ \\
\hline 2. ${ }^{\circ} \mathrm{ESO}$ & $60(12.7)$ & $54(11.5)$ & $114(24.2)$ & $276(10.3)$ & $251(9.4)$ & $527(19.7)$ \\
\hline $3 .^{\circ} \mathrm{ESO}$ & $50(10.6)$ & $49(10.4)$ & $99(21)$ & $292(10.9)$ & $298(11.2)$ & $590(22.1)$ \\
\hline $4 .^{\circ} \mathrm{ESO}$ & $38(8.04)$ & $37(7.9)$ & $75(15.9)$ & $240(9)$ & $232(8.7)$ & $472(17.7)$ \\
\hline Total & $245(51.4)$ & $229(48.6)$ & 474 & $1358(50.8)$ & $1314(49.2)$ & 2672 \\
\hline
\end{tabular}

Fuente: elaboración propia.

\section{Instrumentos}

Para el diseño y validación de la Escala de Convivencia Escolar (véase Apéndice) se tuvieron en cuenta cuestionarios, referentes en la aproximación del constructo, como el Cuestionario de Convivencia Escolar (Ortega \& Del Rey, 2004), los indicadores globales de la convivencia escolar (Martín Babarro, MartínezArias, \& Díaz-Aguado, 2010), el CUVE-R (Álvarez-García, Núñez, Rodríguez, Álvarez, \& 
Dobarro, 2011), el Cuestionario de Clima Social Escolar (Moos \& Trickett, 1974) o el School Development Program School Climate Survey (Haynes et al., 1997).

Asimismo, también han servido de base los programas de mejora de la convivencia, promovidos por las administraciones educativas de España y Latinoamérica (Gijón, 2006; Grisaleña et al., 2005). La prueba inicialmente diseñada constaba de 96 ítems, según el análisis previamente realizado de la literatura científica. Todos los ítems fueron formulados en positivo para facilitar la comprensión y evitar posibles confusiones entre las categorías de respuesta. Los ítems son de tipo Likert de 5 opciones de respuesta, con base en la frecuencia de percepción o de experiencia de los hechos a los que se hace referencia (desde $0=$ nunca, hasta 5 = siempre).

\section{Procedimiento}

Una vez seleccionados los centros, se solicitó permiso a los equipos directivos de cada uno de ellos para realizar la investigación. Cada equipo docente fue informado de los objetivos del estudio así como de la confidencialidad del tratamiento posterior de la información. La recogida de datos fue realizada por los miembros del equipo de investigación. Al entrar en las aulas se informaba al alumnado del carácter anónimo y voluntario de la participación en el estudio.

Tras la codificación de datos, se realizó un análisis factorial exploratorio (AFE) con la muestra piloto, por el que se redujo el número de ítems del instrumento de 96 a 50, al descartar aquellos con índices de discriminación inferiores a 0.3 o con saturaciones factoriales tras la rotación inferiores a 0.3 en todos los factores. Asimismo, se eliminaron los ítems con índices de correlación superiores a 0.85 . Por último, se analizó la legibilidad de los cincuenta ítems para comprobar la idoneidad respecto del grado de complejidad gramatical y semántica de los mismos. Para ello, se introdujo el conjunto de los 50 ítems en el programa INFLESZ v.1.0, que analiza la dificultad del texto a través del Índice de Legibilidad de Flesch-Szigriszt (Barrio-Cantalejo et al., 2008), obteniendo una puntuación de 79.05, lo que indica que en puntuaciones cercanas a 80 o mayores presentan un nivel de comprensión bastante fácil, asequible a población con niveles de educación primaria.

\section{Análisis de datos}

Se llevó a cabo un AFE con la muestra piloto en el que se tuvieron en cuenta los índices de discriminación de los ítems. Con la muestra validación, se realizó un análisis factorial confirmatorio (AFC) y, siguiendo las indicaciones de Hu y Bentler (1999), se estimó el modelo mediante el método robust maximum likehood (RML), debido a que las variables son de naturaleza ordinal e incumplen los supuestos de normalidad. Por ello, para el ajuste del modelo se tuvieron en cuenta los índices: RMSEA: Root Mean Square Error of Approximation; SRMR: Standardized Root Mean Square Residual cuyos valores recomendables son $<0.08$; los índices GFI: Goodness of Fit Index; NNFI: NonNormed Fit Index; RFI: Relative Fit Index; PNFI: Parsimony Normed Fit Index y CFI: Comparative Fit Index, cuyos valores recomendables son $>$ 0.9; y el índice ECVI: Expected Cross-Validation Index, cuyos valores cercanos a cero representan una posibilidad mayor de generalización del modelo. Los análisis se efectuaron con el paquete estadístico LISREL 9.1 (Jöreskog \& Sörbom, 2012), que permite utilizar correlaciones policóricas, cuyo uso es más idóneo en variables con las características señaladas (Jöreskog, 1994).

Por último, se evaluó la fiabilidad mediante los coeficientes alfa de Cronbach y Omega de McDonals, con el paquete estadístico Factor 9.2. (Lorenzo-Seva \& Ferrando, 2006). 


\section{Resultados}

Primer estudio: análisis univariantes y estructura factorial exploratoria

En la Tabla 2 se muestran las medidas de tendencia central, destacando que la desviación estándar supera en todos los ítems el valor de 0.745 , mostrando este resultado que los ítems discriminan verdaderamente (CarreteroDios \& Pérez, 2007). La correlación ítem-total corregida (ri-t) es positiva en todos los ítems, con valores entre 0.248 y 0.876 , indicando que todos contribuyen en la misma dirección a evaluar el constructo teórico al que se hace referencia.

\section{TABLA 2}

Descriptivos univariantes

\begin{tabular}{|c|c|c|c|c|c|c|c|}
\hline Ítem & M & $D E$ & $\begin{array}{l}\text { Asim. } \\
(E . T .= \\
11.2)\end{array}$ & $\begin{array}{l}\text { Curt. } \\
(\text { E.T. }= \\
22.4)\end{array}$ & ri-t & Z & \\
\hline Item1 & 1.36 & 1.184 & $\begin{array}{l}11.27 \\
0.54\end{array}$ & $\begin{array}{c}2.2 .47 \\
-0.512\end{array}$ & 0.394 & 9.24 & $0^{*}$ \\
\hline Item2 & 3.08 & 1.149 & -1.27 & & 0.341 & 15.37 & \\
\hline th & 9 & 1.1 & & & & & \\
\hline Item4 & 0.57 & 0.833 & & 129 & 0.50 & 19.41 & \\
\hline Item5 & 2.61 & 0.991 & -0.305 & -0.404 & 0.762 & 10.91 & $0^{* * *}$ \\
\hline Item6 & 2.89 & 1.056 & .72 & -0.093 & 0.493 & 10.98 & $0^{* * * *}$ \\
\hline Item7 & 1.26 & 1.121 & & 45 & 0.438 & 11.51 & $0^{* * * *}$ \\
\hline Item8 & 2.22 & 1.252 & -0.246 & 91 & 0.422 & 9.43 & $0^{* * 0 *}$ \\
\hline Item 9 & 3.11 & 0.923 & 989 & & 0.649 & 12.99 & $0^{+\infty * t}$ \\
\hline Ítem10 & 1.87 & 1.014 & & -0.325 & 0.468 & 11.02 & $0^{\circ * 0 *}$ \\
\hline Item11 & 1.91 & 1.121 & -0.05 & -0.532 & 0.529 & 10.41 & \\
\hline Item12 & 2.26 & 1.089 & -0.125 & -0.683 & 0.617 & 9.22 & $0^{* * *}$ \\
\hline Item 13 & 2.95 & 0.973 & -0.663 & -0.175 & 0.423 & 10.95 & $0^{* * * *}$ \\
\hline Item 14 & 2.82 & 0.882 & & .282 & 0.424 & 12.35 & $0^{* * *}$ \\
\hline Item 15 & 0.94 & 1.175 & & & 0.634 & 15.78 & $0^{* *+*}$ \\
\hline Item16 & $\begin{array}{l}0.94 \\
0.9\end{array}$ & $\begin{array}{l}1.052 \\
1.052\end{array}$ & & 105 & 0.425 & $\begin{array}{l}13.71 \\
13.78\end{array}$ & $0^{0 * * *}$ \\
\hline & 3.53 & & & & 0.606 & 21.47 & $0^{* * *}$ \\
\hline & 3.48 & 0.7 & & & 0.574 & 18.83 & $0^{n+s *}$ \\
\hline & 4 & 1.0 & & & & .45 & $0^{\circ \ldots *}$ \\
\hline & & 1.2 & & & & & $0^{n+\infty}$ \\
\hline & & & & & & & \\
\hline & 2.48 & & & & & & \\
\hline & 0.55 & & & & & & \\
\hline & 2.63 & 1.0 & & & 0.672 & 11.05 & $0^{* * * * *}$ \\
\hline Item 25 & 3.09 & 0.93 & & & 0.606 & 12.34 & teat \\
\hline Item & 1.79 & 1.1 & & & 0.639 & 9.18 & $0^{* * * *}$ \\
\hline Item & 2.74 & 0.99 & & & 0.553 & 9.83 & $0^{*}$ \\
\hline Item & 2.97 & 0.9 & -0 & 4 & 0.635 & 11.37 & $0^{* * * *}$ \\
\hline Iter & 1.93 & 1.1 & & & 0.574 & 9.42 & $0^{*+*}$ \\
\hline & 2.88 & & & & 0.501 & 11.96 & $0^{+\infty+4}$ \\
\hline & 0.4 & & & & 0.527 & 23.12 & $0^{*+*}$ \\
\hline & 1.06 & & & & 0.694 & 11 & $0^{\circ+\infty}$ \\
\hline & 3.27 & & & & & & $0^{* * n}$ \\
\hline & & & & & & & $0^{\circ * * * *}$ \\
\hline & $\begin{array}{l}3.41 \\
3.40\end{array}$ & & & & & & $0^{* * * *}$ \\
\hline & & & & & & & $0^{* 5 * 4}$ \\
\hline & & 0.9 & & & & & $0^{* * * *}$ \\
\hline & 2.93 & 1.0 & & & & & $0^{\infty \cdots}$ \\
\hline & 3 & 1.0 & & & & & : \\
\hline & 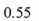 & 0.8 & & & & & \\
\hline & 2. & 1.1 & & & & & \\
\hline & & 0. & & & & & \\
\hline & & & & & & & \\
\hline & & & & & & & \\
\hline & & & & & & & $0^{* \cdots}$ \\
\hline & & & & & & & $0^{\infty \cdots}$ \\
\hline & & & & & & ? & \\
\hline & & & & & & 21.86 & \\
\hline & 2.90 & 0.9 & & -0.052 & 0.511 & 11.4 & $0^{* * *}$ \\
\hline Item50 & 0.50 & 0.789 & 1.711 & 2.889 & 0.523 & 8.32 & $0^{+\infty}$ \\
\hline
\end{tabular}

Las respuestas en la muestra piloto no presentan una distribución normal (Tabla 2), puesto que los índices de asimetría y curtosis obtenidos y la prueba de Kolmogorov-Smirnov, así lo refleja. También se puede observar que la mayor parte de los índices en la curtosis son negativos, mostrando puntuaciones dispersas y situadas por debajo de la curva de la distribución normal.

Asimismo, el índice multivariado de curtosis o coeficiente de Mardia tiene un valor de 3044.278, siendo lo recomendable valores inferiores a 70 (Mardia, 1970). Consecuentemente, se optó por utilizar el método RML en el AFE de la muestra piloto, ya que no requiere una distribución normal de la muestra y con muestras superiores a 200 sujetos, como es el caso de esta investigación, no presenta diferencias significativas para la idoneidad en la estimación del ajuste del modelo o solución factorial (Bandalos, 1993). La idoneidad del análisis factorial se confirmó calculando el índice de Kaiser-Meyer-Olkin y la prueba de esfericidad de Bartlett $(\mathrm{KMO}=0.917$; $\left.\chi^{2}=11013.3 ; g l=1225 ; p=0\right)$. En este análisis factorial preliminar se utilizó el método rotación de extracción oblicua, PROMAX (valor Kappa = 4), del que surgieron ocho factores que explican un $63 \%$ de la varianza (Tabla 3). 
TABLA 3

Solución factorial

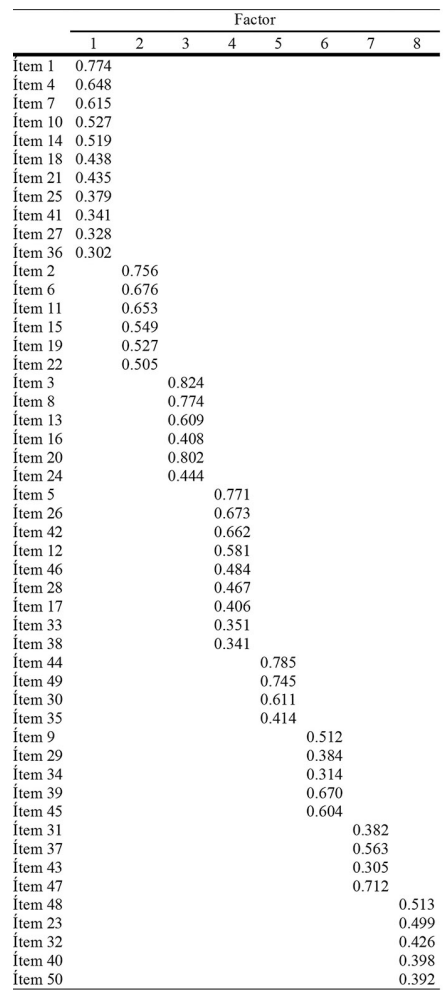

Fuente: elaboración propia.

Teniendo en cuenta la composición de cada factor, se denominó al primero Gestión interpersonal positiva (29 \%), al segundo Victimización (8\%), al tercero Disruptividad (7 $\%)$, al cuarto Red social de iguales (6\%), al quinto Agresión (3\%), al sexto Ajuste normativo (2\%), al séptimo Indisciplina (2\%) y al octavo Desidia docente (2\%).

\section{Segundo estudio: análisis factorial confirmatorio}

Para comprobar el ajuste del modelo obtenido con el AFE, se realizó un AFC con la muestra validación $(n=2672)$ siguiendo el mismo modelo de estimación RML, puesto que el análisis de la curtosis multivariante con esta muestra adquiere un valor de 420.48. Así, los índices de ajuste obtenidos muestran valores de: $\chi^{2}=3489.84 ; \mathrm{RMSEA}=0.05 ; \mathrm{SRMR}=0.05 ;$ $\mathrm{CFI}=0.96 \mathrm{GFI}=0.95 ; \mathrm{NNFI}=0.96$ y $\mathrm{RFI}=$ 0.95 .
Posteriormente, teniendo en cuenta que prácticamente la totalidad de los estudios de convivencia se han realizado con muestras de alumnado de secundaria, se procedió a realizar un AFC multigrupo con el fin de comprobar el ajuste del modelo para el alumnado de educación primaria y secundaria. Se evalúo la invarianza factorial del AFC en función del ciclo educativo de los adolescentes, con el objeto de verificar si su ajuste difería en la educación primaria, primer ciclo de educación secundaria y segundo ciclo de educación secundaria. Para llevar a cabo este análisis, se estimaron dos modelos que se compararon a través del test de la diferencia en $\chi^{2}$ . Los modelos fueron los siguientes: a) un primer modelo multigrupo que no impone ninguna igualdad entre las saturaciones y las correlaciones entre factores (modelo sin restringir o modelo de línea base) y b) un segundo modelo que impone la igualdad entre todas las saturaciones y las correlaciones entre los factores en los grupos (modelo restringido). A continuación, se examinó la diferencia entre los valores de $\chi^{2}$ y grados de libertad de los dos modelos para poder compararlos en términos de ajuste.

En la Tabla 4 se presenta un resumen de los índices de ajuste multigrupo obtenidos del test de la diferencia en $\chi^{2}$ (Satorra \& Bentler, 2001). La diferencia en el valor de $\chi^{2}$ entre el modelo restringido y sin restringir no fue significativa $\left(\Delta_{\chi}^{2}(256)=16194.897, p>0.05\right)$, lo que es indicativo de una invarianza métrica, que señala la existencia de equivalencia en el ajuste del modelo en los tres ciclos educativos sometidos a validación.

TABLA 4

Prueba de Satorra-Bentler

\begin{tabular}{lrrrrrrr}
\hline Model & DF & $\chi^{2}$ & P & NFI & IFI & RFI & TLI \\
\hline Model 1 & 256 & 16194.897 & 0.08 & 0.096 & 0.097 & 0.094 & 0.094 \\
Model 2 & 256 & 16194.897 & 0.08 & 0.096 & 0.097 & 0.094 & 0.094 \\
\hline
\end{tabular}

Fuente: elaboración propia

Por último, se realizaron los análisis de fiabilidad del cuestionario, hallando un valor total de alfa de Cronbach de $=0.94 \mathrm{y}$ de Omega de Mcdonald de $=0.94$ y en las dimensiones un alfa de Cronbach de: Gestión 
interpersonal positiva $=0.83$; Victimización $=$ 0.9; Disruptividad $=0.9$; Red social de iguales $=$ 0.78 ; Agresión $=0.89$; Ajuste normativo $=0.88$; Indisciplina $=0.86$ y Desidia docente $=0.92$.

\section{Discusión}

El objetivo de esta investigación ha sido desarrollar y validar un instrumento para describir la convivencia escolar desde la percepción del alumnado de educación primaria y educación secundaria. Según los resultados, la convivencia escolar se compone de ocho factores, positivos y negativos, de distinto grado de relevancia que se han denominado: gestión interpersonal positiva, victimización, disruptividad, red social de iguales, agresión, ajuste normativo, indisciplina y desidia docente. El factor más importante del constructo convivencia ha resultado ser la forma en la que los profesores gestionan las relaciones interpersonales en el centro educativo. Aludiendo a la conducta social ejemplar, la buena comunicación con el alumnado y sus familias y a la atención de las dificultades que estos pueden encontrar. Esta dimensión registra la calidad en las relaciones que los docentes establecen con su alumnado y las familias de este. Una dimensión que incluye el conjunto más importante, a su vez, de lo que se conoce como clima escolar (Bear et al., 2011; Moos \& Moos, 1978; Yang et al., 2013), en la medida en que es coherente con lo que investigaciones previas han demostrado, que favorece el clima social y presenta efectos positivos sobre el ajuste psicológico de los escolares en general (Kuperminc, Leadbeater, \& Blatt, 2001; Roeser $\&$ Eccles, 1998) y particularmente de aquellos que pueden tener riesgos de dificultades académicas, emocionales o de conflictos (Haynes et al., 1997). Esta dimensión se refiere a la verticalidad de la convivencia que describe la conducta que los escolares son capaces de apreciar en la profesionalidad de sus docentes y, a tenor de los resultados, consideran relevante en su percepción de la convivencia. Estas características que los docentes expresan y su alumnado registra se han mostrado en diferentes modelos educativos y culturas como unos de los aspectos que definen la convivencia escolar (Yang et al., 2013). Pero la relevancia de dicha verticalidad del profesorado al alumnado no solo parece ser clave en términos positivos, sino también negativos. Así, se ha encontrado un factor, aun con un impacto mucho menor, que refleja lo que se ha denominado la desidia, el desinterés docente o su incompetencia profesional, observada por el alumnado. La nula o baja percepción del alumnado relativa al interés y el apoyo del profesorado no es un aspecto muy abordado en la literatura científica (Comellas, 2010; Olson \& Wyett, 2000), pero con base en los resultados, debería ser una aportación para tener en cuenta en la evaluación de la convivencia escolar. Los escolares reconocen el impacto positivo que tiene la buena gestión del docente hacia ellos y hacia las actividades escolares, pero también señalan un factor negativo en los docentes que no cumplen las expectativas de ser buenos administradores de la vida en común, y que claramente no favorece que estos tengan una buena percepción de la convivencia escolar (Otienoh, 2011).

Pero esta verticalidad no define en exclusividad a la convivencia escolar, sino que las relaciones entre los iguales son también muy importantes. Es lo que se ha llamado la horizontalidad de la convivencia escolar. La disruptividad y el impacto de ser victimizado por otros se muestran muy relevantes para el buen ajuste, fiabilidad y validez del instrumento objeto de análisis, junto a la conducta agresiva y la indisciplina. Son cuatro dimensiones las que hay que formular en términos negativos y que parecen consustanciales en la definición de convivencia, porque la perturban (Astor, Benbenishty, Zeira, \& Vinokur, 2002; Bear et al., 2011; Pulido, Martin, \& Lucas, 2011). Debe destacarse que el factor que más impacta en este orden horizontal de la convivencia es la victimización. Ser maltratado, atacado, golpeado o, en definitiva, victimizado, ha sido el problema de la convivencia escolar más ampliamente investigado en los últimos años (Casas, Del Rey, \& Ortega-Ruiz, 2013; Baldry \& Farrington, 2007; 
Smith et al., 2012; Ttofi \& Farrington, 2011). Estos resultados refrendan la larga trayectoria de estudios que muestran hasta qué punto se mejora el clima y la convivencia escolar cuando se reduce la victimización (Ttofi \& Farrington, 2011; Williams \& Guerra, 2007).

En el lado positivo, aunque con menor importancia estadística, está el ajuste social entre iguales y las buenas relaciones entre compañeros, en consonancia con muchos de los estudios sobre convivencia que resaltan el valor del compañerismo y el ajuste a las normas sociales como un factor de prevención del riesgo de violencia escolar (Buelga, Cava, \& Musitu, 2012). Estos resultados avalan lo señalado en múltiples estudios anteriores (Comellas, 2010; Gazquez et al., 2011; Godás Otero et al., 2008; Molloy, Gest, \& Rulison, 2011) que consideran las relaciones entre los iguales como un foco relevante en la evaluación de la convivencia escolar.

En síntesis, los ocho factores que componen este instrumento recogen la percepción del alumnado sobre la convivencia escolar, en consonancia con una parte importante de los estudios sobre esta temática (Álvarez-García, et al., 2011; Bear et al., 2011; Bradshaw et al., 2009; Gazquez et al., 2011). Por ello, podríamos hablar de un instrumento que integra gran parte de las claves conceptuales, desde las que ha sido observado este poliédrico y multidimensional constructo que denominamos convivencia escolar. Puede decirse que tiene dos grandes planos: la horizontalidad en la que importa mucho no ser victimizado, estar ajustado a las normas sociales compartidas y a las reglas disciplinares percibidas como positivas; y la verticalidad, en la que es importante percibir que los docentes se ocupan con actitudes profesionales positivas y seguras, las necesidades de los escolares y la buena comunicación con las familias.

Lo analizado es válido tanto para la convivencia en secundaria como en primaria. Se evidencia así una cierta universalidad de estos ocho factores que es reseñable. La convivencia escolar precisa actualmente de instrumentos con propiedades psicométricas contrastadas, que tengan en cuenta las limitaciones de las investigaciones en ciencias sociales y que asuman las características de la metodología empleada actualmente en la investigación psicopedagógica (Campo-Arias \& Oviedo, 2008). Dicho lo anterior, no hay duda de que este trabajo presenta ciertas limitaciones que abren, a su vez, futuras líneas de investigación. A pesar de utilizar una amplia muestra, esta no es representativa del país, lo que permitiría afirmar que la validez del constructo es para toda la población escolar española, y la validez se ha realizado con población estudiantil y sería relevante contar, igualmente, con muestras de profesorado y familias que permitieran disponer de la imagen completa de los protagonistas de la convivencia escolar.

\section{Referencias}

Álvarez-García, D., Núñez, J. C., Rodríguez, C., Álvarez, L., \& Dobarro, A. (2011). Propiedades psicométricas del Cuestionario de Violencia Escolar - Revisado (CUVER). Revista de Psicodidáctica/Journal of Psychodidactics, 16(1). Recuperado de http://www.ehu.es/ojs/index.php/psicodi dactica/article/view/1146

Astor, R. A., Benbenishty, R., Zeira, A., \& Vinokur, A. (2002). School climate, observed risky behaviors, and victimization as predictors of high school students' fear and judgments of school violence as a problem. Health Education Eु Behavior, 29(6), 716-736. doi:10.1177/109019802237940

Baldry, A. C., \& Farrington, D. P. (2007). Effectiveness of programs to prevent school bullying. Victims and Offenders, 2(2), 183-204.

Bandalos, D. L. (1993). Factors influencing cross-Validation of Confirmatory Factor Analysis Models. Multivariate Behavioral Research, 28(3), 351-374. doi:10.1207/ s15327906mbr2803 3

Barrio-Cantalejo, I. M., Simón-Lorda, P., Melguizo, M., Escalona, I., Marijuán, M. I., 
\& Hernando, P. (2008). Validación de la Escala INFLESZ para evaluar la legibilidad de los textos dirigidos a pacientes. Anales del Sistema Sanitario de Navarra, 31(2), 135-152.

Bear, G. G., Gaskins, C., Blank, J., \& Chen, F. F. (2011). Delaware School Climate SurveyStudent: Its factor structure, concurrent validity, and reliability. Journal of School Psychology, 49(2), 157-174. doi:10.1016/ j.jsp.2011.01.001

Bibou-Nakou, I., Tsiantis, J., Assimopoulos, H., Chatzilambou, P., \& Giannakopoulou, D. (2012). School factors related to bullying: A qualitative study of early adolescent students. Social Psychology of Education, 15, 125. doi:10.1007/s11218-012-9179-1

Bradshaw, C. P., Debnam, K., Koth, C. W., \& Leaf, P. (2009). Preliminary validation of the implementation phases inventory for assessing fidelity of schoolwide positive behavior supports. Journal of Positive Behavior Interventions, 11(3), 145-160. doi: $10.1177 / 1098300708319126$

Buelga, S., Cava, M. J., \& Musitu, G. (2012). Reputación social, ajuste psicosocial y victimización entre adolescentes en el contexto escolar. Anales de Psicología, 28(1), 180-187. doi:10.6018/ analesps.28.1.140652

Burke, M. D., Davis, J. L., Lee, Y. H., Hagan-Burke, S., Kwok, Oi-man, \& Sugai, G. (2012). Universal screening for behavioral risk in elementary schools using SWPBS expectations. Journal of Emotional and Behavioral Disorders, 20(1), 38-54. doi: $10.1177 / 1063426610377328$

Campo-Arias, A., \& Oviedo, H. C. (2008). Psychometric properties of a scale: Internal consistency. Revista de Salud Pública, 10(5), 831-839.

Carretero-Dios, H., \& Pérez, C. (2007). Standards for the development and review of instrumental studies: Considerations about test selection in psychological research. International Journal of Clinical and Health Psychology, 7(3), 863-882.
Casas, J. A., Del Rey, R., \& Ortega-Ruiz, R. (2013). Bullying and cyberbullying: Convergent and divergent predictor variables. Computers in Human Behavior, 29(3), 580-587. http://doi.org/10.1016/j.ch b.2012.11.015

Caso, J., Salgado, B., Rodríguez, J. C., Contreras, L. A., \& Urías, E. (2010). Propiedades psicométricas de la Escala de Clima Escolar para adolescentes. Ensenada, México: Universidad Autónoma de Baja California.

Comellas, M. J. (2010). The role of teachers in the prevention of violence of education centres. Psychology, Society, \& Education, 2(1), 39-53.

Delors, J. (1996). Learning: The treasure within. París: Unesco.

Fullan, M., \& Hargreaves, A. (1992). Teacher development and educational change. London: Routledge.

García Raga, L., \& López Martín, R. (2011). Convivir en la escuela. Una propuesta para su aprendizaje por competencias. Revista de Educación, 356, 531-555.

Gázquez, J. J., Cangas, A. J., Perez-Fuentes, M. C., Padilla, D., \& Cano, A. (2007). Perception of the school violence by the relatives: A comparative study in four European countries (RID A-5497-2009). International Journal of Clinical and Health Psychology, 7(1), 93-105.

Gázquez, J. J., Pérez, M. C., \& Carrión, J. J. (2011). Clima escolar y resolución de conflictos según el alumnado: un estudio europeo. Revista de Psicodidáctica, 16(1), $39-58$.

Gijón, J. (2006). La convivencia escolar como innovación: un análisis sobre el caso andaluz utilizando el "Concerns-Based Adoption Model". Education Policy Analysis Archives/Archivos Analíticos de Políticas Educativas, 14, 1-22.

Godás Otero, A., Santos Rego, M. A., \& Lorenzo, M. del M. (2008). Convivir en los centros educativos. ¿Cómo lo ven los alumnos, padres y profesores? Bordón. Revista de Pedagogía, 60(1), 41-58. 
Grisaleña, J., Fernández, A., \& Campo, A. (2005). La convivencia en los centros de secundaria: un estudio de casos. Revista Iberoamericana de Educación, 38, 121-145.

Hair, J. F., Black, B., Babin, B., Anderson, R. E., \& Tatham, R. L. (2005). Multivariate data analysis (6a. ed.). New Jersey: PrenticeHall.

Haynes, N. M., Emmons, C., \& Ben-Avie, M. (1997). School climate as a factor in student adjustment and achievement. Journal of Educational and Psychological Consultation, 8(3), 321-329. doi:10.1207/ s1532768xjepc0803 4

Hu, L., \& Bentler, P. M. (1999). Cutoff criteria for fit indexes in covariance structure analysis: Conventional criteria versus new alternatives. Structural Equation Modeling: A Multidisciplinary Journal, 6(1), 1-55. doi:10.1080/10705519909540118

Jöreskog, K. G. (1994). On the estimation of polychoric correlations and their asymptotic covariance matrix. Psychometrika, 59(3), 381-389. doi:10.1007/ BF02296131

Jöreskog, K. G., \& Sörbom, D. (2012). Lisrel 9.1. Skokie: Scientific Software International, Inc. Recuperado de http://www.ssicentral.c om/lisrel/

Kuperminc, G. P., Leadbeater, B. J., \& Blatt, S. J. (2001). School social climate and individual differences in vulnerability to psychopathology among middle school students. Journal of School Psychology, 39(2), 141-159. doi:10.1016/ S0022-4405(01)00059-0

Kuperminc, G. P., Leadbeater, B. J., Emmons, C., \& Blatt, S. J. (1997). Perceived school climate and difficulties in the social adjustment of middle school students. Applied Developmental Science, 1(2), 76-88. doi:10.1207/s1532480xads0102_2

Lorenzo-Seva, U., \& Ferrando, P. J. (2006). FACTOR: A computer program to fit the exploratory factor analysis model. Behavior Research Methods, 38(1), 88-91.

Mardia, K. V. (1970). Measures of multivariate skewness and kurtosis with applications.
Biometrika, 57(3), 519-530. doi:10.1093/ biomet/57.3.519

Martín-Babarro, J., Martínez-Arias, R., \& DíazAguado, M. J. (2010). Estudio estatal sobre la convivencia escolar. Madrid: Ministerio de Educación.

McIntosh, K., MacKay, L. D., Andreou, T., Brown, J. A., Mathews, S., Gietz, C., \& Bennett, J. L. (2011). Response to intervention in Canada: Definitions, the evidence base, and future directions. Canadian Journal of School Psychology, 26(1), 18-43. doi:10.1177/0829573511400857

Mitchell, M. M., Bradshaw, C. P., \& Leaf, P. J. (2010). Student and teacher perceptions of school climate: A multilevel exploration of patterns of discrepancy. Journal of School Health, 80(6), 271-279. doi:10.1111/ j.1746-1561.2010.00501.x

Molloy, L. E., Gest, S. D., \& Rulison, K. L. (2011). Peer influences on academic motivation: Exploring multiple methods of assessing youths' most "influential" peer relationships. The Journal of Early Adolescence, 31(1), 13-40. doi: $10.1177 / 0272431610384487$

Moos, R. H., \& Moos, B. S. (1978). Classroom social climate and student absences and grades. Journal of Educational Psychology, 70, 263-269. Recuperado de http://www.eric.ed.gov/ERICWebPortal $/$ detail? accno $=\mathrm{EJ} 188001$

Moos, R. H., \& Trickett, E. J. (1974). Classroom environment scale manual. Palo Alto: Consulting Psychologist Press.

Morata-Ramírez, M. de los Á., \& Holgado-Tello, F. P. (2013). Construct validity of Likert scales through confirmatory factor analysis: A simulation study comparing different methods of estimation based on Pearson and polychoric correlations. International Journal of Social Science Studies, 1(1), 54-61. doi:10.11114/ijsss.v1i1.27

Olson, C. O., \& Wyett, J. L. (2000). Teachers need affective competencies. Education, 120(4), 741-744.

Ortega, R., \& Del Rey, R. (2004). Construir la Convivencia. Barcelona: Edebé. 
Ortega, R., Del Rey, R., \& Sanchez, V. (2012). Nuevas dimensiones de la convivencia escolar y juvenil. Ciberconducta y relaciones en la Red: Ciberconvivencia. Madrid: Ministerio de Cultura, Educación y Deporte.

Otienoh, R. O. (2011). Teachers' lack of deeper analytical reflections: Who is to blame? Reflective Practice, 12(6), 733-747. doi:10.1080/14623943.2011.601091

Pulido, R., Martin, G., \& Lucas, B. (2011). Risk profiles and peer violence in the context of school and leisure time. Spanish Journal of Psychology, 14(2), 701-711. doi:10.5209/ rev SJOP.2011.v14.n2.18

Roeser, R. W., \& Eccles, J. S. (1998). Adolescents' perceptions of middle school: Relation to longitudinal changes in academic and psychological adjustment. Journal of Research on Adolescence, 8(1), 123-158.

Satorra, A., \& Bentler, P. M. (2001). A scaled difference chi-square test statistic for moment structure analysis. Psychometrika, 66(4), 507-514. doi:10.1007/BF02296192

Smith, P. K., Kupferberg, A., Mora-Merchan, J. A., Samara, M., Bosley, S., \& Osborn, R. (2012). A content analysis of school anti-bullying policies: A follow-up after six years. Educational Psychology in Practice, 28(1), 47-70. doi: 10.1080/02667363.2011.639344

Ttofi, M. M., \& Farrington, D. P. (2011). Effectiveness of school-based programs to reduce bullying: A systematic and metaanalytic review. Journal of Experimental Criminology, 7(1), 27-56.

Viguer, P., \& Avià, S. (2009). Un modelo local para la promoción de la convivencia y la prevención de la violencia entre iguales desde el ámbito comunitario. Cultura y Educación, 21(3), 345-359. doi:10.1174/113564009789052334

Williams, K. R., \& Guerra, N. G. (2007). Prevalence and predictors of internet bullying. Journal of Adolescent Health, 41(6), S14-S21. doi:10.1016/ j.jadohealth.2007.08.018

Yang, C., Bear, G. G., Chen, F. F., Zhang, W., Blank, J. C., \& Huang, X. (2013). Students' perceptions of school climate in the U.S. and China. School Psychology Quarterly, 28(1), 7-24. doi:10.1037/spq0000002

\section{APÉNDICE}

\section{Escala de Convivencia Escolar}

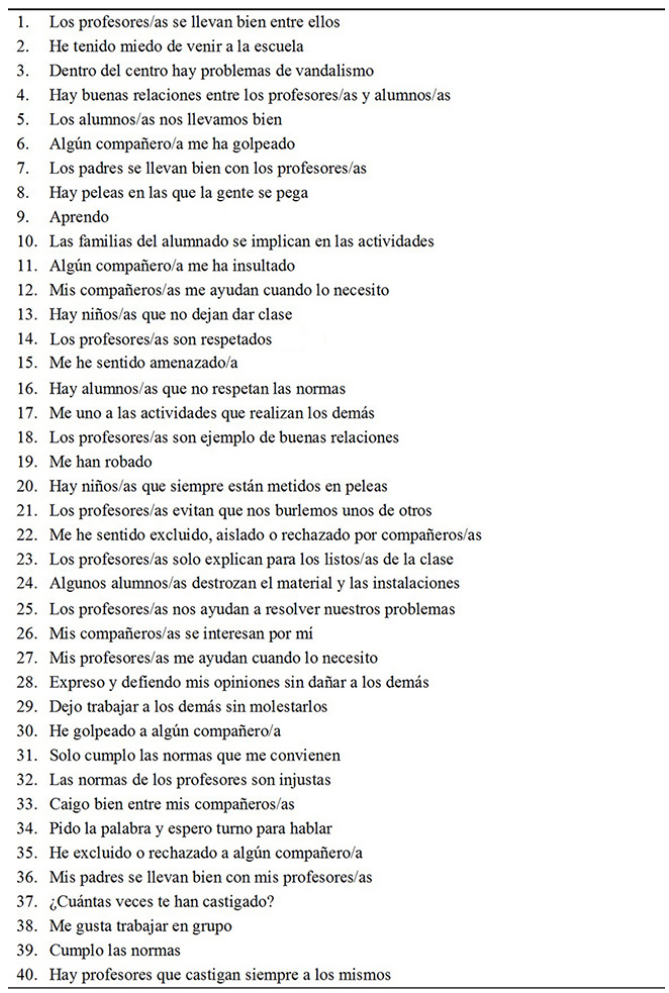

\section{Notas}

* Artículo de investigación. 